॥ Wir Neurologen wollen unser Fach zusammenhalten

Prof. Dr. Peter Berlit

Deutsche Gesellschaft für Neurologie (DGN)

Reinhardtstr. 27C, 10117 Berlin, Deutschland

berlit@dgn.org

\title{
Die Rubrik „Im Fokus“
}

Vermutlich wurde es schon in der letzten Ausgabe bemerkt: die DGNeurologie hat eine neue Rubrik. Neben den verschiedenen Original- und Übersichtsarbeiten sowie unseren Gesellschaftsseiten gibt es jetzt die Rubrik „Im Fokus“. In ihr wollen wir aktuelle Themen darstellen, die für die Neurologie von besonderer Bedeutung sind. Im letzten Heft war es die Bestandsaufnahme unserer Kommission für Anhaltszahlen. In diesem Heft widmen sich unsere jungen Neurologen dem Thema Weiterbildung in Europa.

Es ist interessant, dass in den „Vereinigten Staaten von Europa" die neurologische Weiterbildung nicht ganz so unterschiedlich gehandhabt wird, wie dies mit der Sprache und kulinarisch der Fall ist. Dabei nimmt Deutschland insofern eine Sonderstellung ein, als wir das einzige Land sind, in dem 1 Jahr Weiterbildung in der Psychiatrie Pflicht ist. Eine psychiatrische Weiterbildung wird auch in anderen europäischen Ländern angeboten, ist aber deutlich kürzer und v. a. fakultativ. In den meisten anderen Staaten ist es die innere Medizin, die einen hohen Stellenwert hat.

Und wir müssen uns in Deutschland fragen, wie wir in der vorgesehenen Weiterbildungszeit von 4 Jahren Neurologie und 1 Jahr Psychiatrie alle Inhalte vermitteln und lernen wollen, die für den Facharzt gefordert sind. Mit der zunehmenden Diversifizierung unseres Fachs wird das Wissen ja nicht weniger, sondern kontinuierlich mehr, deutlich mehr! Wenn wir nur den enormen Wissenszuwachs im Bereich der Neuroimmunologie betrachten, wer soll das alles kompetent wissen? Und wir haben die peripheren Nerven, das Rückenmark, die Neuropsychologie, die Bewegungsstörungen und den Schlaganfall. Es sind ja nicht nur theoretische Dinge, die wir in unserem Fach beherrschen müssen, sondern zunehmend auch praktische Fertigkeiten, die verlangt werden. Und ich denke, wir sind uns alle einig, dass wir unser Fach zusammenhalten wollen. Wir wollen nicht, wie in der inneren Medizin, einen Facharzt für Schlaganfallmedizin, einen Facharzt für Bewegungsstörungen, einen Facharzt für Epileptologie und einen Facharzt für Neuroonkologie. Dass in der Wissenschaft eine Spezialisierung erforderlich ist, ist unstrittig. Der wissenschaftliche Universalist ist heute nicht mehr möglich.

DGNeurologie 2019 • 2 (4): 241

https://doi.org/10.1007/s42451-019-0094-y

๑) Springer Medizin Verlag GmbH, ein Teil von Springer Nature 2019
Aber als Neurologin und Neurologe in Klinik und Praxis müssen wir das Fach weiterhin in seiner ganzen Breite überschauen und unseren Kunden, den Patienten, kompetent zur Seite stehen. Schlaganfallmedizin erfordert viel kardiologisches und metabolisches Grundwissen. Für Neuroimmunologie und Neuroonkologie ist die Kenntnis der Immun- und Chemotherapeutika mit ihren Nebenwirkungen und Risiken unabdingbar. Alle diese Inhalte sind eng mit der inneren Medizin verzahnt. Internistische Grundkenntnisse sind daher für den Neurologen essenziell.

Und wie verhält es sich mit der Psychiatrie? Ich selbst habe mein Psychiatriejahr auf der Akutstation und in der Gerontopsychiatrie absolviert und war zu einem großen Teil dort eingesetzt, wo neurologisch-intensivmedizinisches Wissen gefordert war. Oft hatte ich den Eindruck, dass ich die Kolleginnen und Kollegen in der Psychiatrie bei allen „organischen Problemen“ unterstützen musste. Und mein psychiatrisches Grundwissen erlernte ich überwiegend in der Neurologie, bei der Betreuung von Patienten mit Verwirrtheitszuständen, Delir und kognitiven Störungen. Hinzu kommen die depressiven Begleitsymptome bei vielen neurologischen Erkrankungen und natürlich die somatoformen Störungen, die sich sehr häufig mit neurologischen Symptomen äußern. Ich denke, wir werden weiter das Gespräch mit unseren psychiatrischen Kollegen suchen und gemeinsam überlegen müssen, wie die Facharztweiterbildung in Zukunft sinnvoll zu gestalten ist. Es ist wichtig, dass wir all jene Fähigkeiten, Fertigkeiten und Kenntnisse in den 5 Jahren Facharztweiterbildung tatsächlich erwerben können, die wir als Neurologinnen und Neurologen benötigen. Dafür müssen zeitliche Freiräume geschaffen werden, nicht nur in der Psychiatrie, sondern auch in der inneren Medizin und Neuroradiologie. Hierbei können wir in Deutschland etwas von Europa lernen. Was meinen Sie? Schreiben Sie mir!

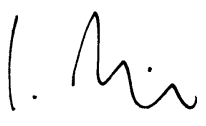

Ihr Peter Berlit

Interessenkonflikt. P. Berlit gibt an, dass kein Interessenkonflikt besteht. 